INTERVENTIONAL CARDIOLOGY AND SURGERY

\title{
Clinical and angiographic outcome after conventional angioplasty with optional stent implantation compared with direct stenting without predilatation
}

\author{
S Miketic, J Carlsson, U Tebbe
}

Heart 2002;88:622-626

\begin{abstract}
Objective: To compare in a randomised trial the procedural and clinical outcome and long term patency of conventional angioplasty with optional stent implantation versus direct stenting without predilatation.

Methods: Patients undergoing coronary intervention for symptomatic coronary artery disease were randomly assigned to conventional angioplasty with optional stenting or to direct stent implantation without predilatation. The post-stent treatment consisted of antiplatelets. Follow up angiography was performed six months after the initial procedure.

Results: Between December 1998 and August 1999, 181 of 190 eligible patients were randomly assigned to either optional stenting $(n=92)$ or direct stenting $(n=89)$. The procedural success was similar in both groups $(87(97.8 \%)$ in the optional $v 87(94.6 \%)$ in direct stenting group, $p=0.88)$. There were five cases of crossover from the direct stenting to the optional stenting group. Six patients experienced a $Q$ wave myocardial infarction without further complications ( 4 in the optional $v 2$ in the direct stenting group, $p=0.36$ ). One patient in whom coronary angioplasty failed underwent elective bypass surgery. No patients required urgent bypass surgery and no patients died. The reduction in late luminal loss (mean (SD) 1.19 $(0.87) \mathrm{mm}$ in the optional $v 0.62(0.69) \mathrm{mm}$ in the direct stenting group, $p=0.004$ ) led to a significant improvement in minimal luminal diameter at follow up $(1.87(0.93) \mathrm{mm}$ in the optional $v$ $2.56(0.86) \mathrm{mm}$ in the direct stenting group, $p=0.002)$, resulting in a significant reduction in restenosis rate, defined as $>50 \%$ diameter stenosis at follow up 6.5 (2.1) months after the initial procedure $(28(30.4 \%)$ in the optional $v 14(15.7 \%)$ in the direct stenting group, $p=0.019)$. Direct stenting significantly reduced the overall procedure and fluoroscopy times, the amount of contrast medium used, and the number of angioplasty catheters needed. The incidence of clinical events during the six month follow up did not differ significantly between the groups. No patient died during follow up.

Conclusions: Direct stent implantation without predilatation significantly reduced late luminal loss, giving a better improvement in minimal luminal diameter and restenosis rate than with optional stenting. There were five patients in whom direct stenting failed who needed predilatation followed by stent implantation. However, in most patients direct stent implantation without predilatation is a feasible treatment option with a favourable long term outcome and a low incidence of complication. The procedure may help to reduce the cost of coronary interventions by reducing overall procedure and fluoroscopy times, the amount of contrast medium used, and the number of angiography catheters needed.
\end{abstract}

See end of article for authors' affiliations

Correspondence to: Dr S Miketic, Medizinische Klinik II, Klinikum Lippe-GmbH,

Roentgenstraße 18 D-32756 Detmold, Germany;

miketic@freenet.de

Accepted 12 June 2002

$\mathrm{T}$ he use of intracoronary stents in patients undergoing percutaneous coronary intervention continues to increase such that stents are being used in $50-70 \%$ of procedures worldwide. ${ }^{1}$ Progress in technology and improvement in stent design, with a lower stent profile and greater flexibility, have enabled the placement of second generation stents without predilatation..$^{2-4}$ Experimental studies have shown that intimal hyperplasia seems to be the cellular response to shear stress induced endothelial deformation ${ }^{5}$; therefore, the technique of direct stent implantation has the potential to reduce vessel wall injury compared with the conventional procedure with balloon dilatation followed by optional stent placement. ${ }^{6}$ Retrospective analysis has confirmed the feasibility of this procedure ${ }^{7}$ but randomised data are not yet available.

The aim of the present randomised study was to compare conventional angioplasty with optional stenting versus direct stent implantation without predilatation.

\section{METHODS}

Patient selection

Patients scheduled for coronary angioplasty with an angiographically proven, functionally significant narrowing of one de novo lesion in a major native coronary artery with a vessel diameter $\geqslant 2.75 \mathrm{~mm}$ were considered for inclusion. Patients with a totally occluded target vessel, left main or bifurcational stenosis, and very tortuous or angulated vessels and patients with acute coronary syndromes requiring angioplasty were excluded. Coronary intervention was performed according to the guidelines of the American Heart Association/American College of Cardiology. ${ }^{8}$

The study was carried out according to the principles of the Declaration of Helsinki. Written informed consent according

Abbreviations: BENESTENT, Belgium Netherlands stent study; CABG, coronary artery bypass grafting; CPK, creatine phosphokinase; STRESS, stent restenosis study; TIMI, thrombolysis in myocardial infarction 
to local practice for the intervention as described above and for a follow up angiography six months after the procedure was obtained for every patient.

\section{Randomisation}

Permeated block randomisation was used to maintain chronological balance in the number of patients assigned to the two treatment strategies.

\section{Angioplasty procedure}

All procedures were done with a large lumen 6 French gauge guiding catheter (Ascent, Medtronic Interventional Vascular, Kerkrade, Netherlands) and a femoral approach. The diameters of the normal appearing segments proximal and distal to the target lesion were averaged to determine the balloon size. All patients received $100 \mathrm{mg}$ oral aspirin daily before and 10000 IU heparin at the start of the procedure. The femoral sheath was removed after normalisation ( $<40$ seconds) of the partial thromboplastin time.

\section{Conventional angioplasty with optional stenting}

Patients assigned to conventional angioplasty with optional stenting received one or more dilatations followed by optional stent implantation to achieve a residual stenosis $<15 \%$. The interim angiographic success was calculated by on line quantitative coronary analysis during the intervention. The procedure was terminated if the residual stenosis was below $15 \%$ and no visible dissection with compromised flow was present.

\section{Direct stent implantation without predilatation}

In patients assigned to direct stent implantation the target lesion was crossed with an ACS MultiLink Duet stent (Guidant SA, Zaventem, Belgium). The MultiLink Duet is mounted on a low profile, high pressure (rated burst $16 \mathrm{~atm}$ ) flexible balloon delivery system. The dimension of the implanted stents was determined to completely cover the lesion. After deployment of the stent inside the target lesion, the stent was expanded under a high pressure of at least $12 \mathrm{~atm}$. If crossing of the target lesion with the stent delivery system did not succeed, the patient was reallocated to the conventional angioplasty group. Immediately after stent implantation additional antithrombotic treatment with clopidogrel $75 \mathrm{mg}$ once daily was started and continued for three weeks. Aspirin was continued indefinitely.

\section{Angiographic analysis}

Three angiograms in at least two orthogonal views showing the worst projection were obtained for each patient: one before the intervention, one immediately after, and one at follow up six months later. Intracoronary glyceryl trinitrate $(200 \mu \mathrm{g})$ was injected before all angiographic assessments. Quantitative analysis with a validated edge detection system (DCI, Philips Medical Systems, Best, Netherlands) ${ }^{9}$ was performed on line during the procedure and routinely before, after, and at follow up. Angiograms at follow up were evaluated off line by two senior cardiologists not personally involved in the angioplasty procedure of the study patients. The treated segment at follow up was determined by exact comparison with the prior intervention. The diameter of the normal segment proximal to the treated area in at least two orthogonal views was averaged to determine the reference diameter.

\section{Definition and clinical follow up}

Angiographic success was defined as $<15 \%$ residual diameter stenosis of the dilated artery in the optimal angioplasty group and $<10 \%$ residual diameter stenosis in the direct stent implantation group. Adverse clinical events were as follows: (a) acute or threatened vessel occlusion with compromised antegrade flow of the target vessel (TIMI (thrombolysis in myocardial infarction) grade $\leqslant 2$ ) complicating angioplasty and requiring repeat angioplasty or stent implantation; (b) elective or emergency coronary artery bypass grafting (CABG); (c) myocardial infarction, defined as chest pain lasting > 30 minutes, new $\mathrm{Q}$ waves lasting 0.04 seconds or more, or ST segment elevation $(>1 \mathrm{mV})$ in at least two contiguous leads on the ECG measured immediately after the procedure and 6 and 24 hours later, and a more than twofold increase in creatine phosphokinase (CPK) above baseline after 6, 12, and 24 hours; and (d) cardiac death. All patients were followed up for at least six months after discharge.

\section{End points}

The primary end points were the restenosis rate (defined as $>50 \%$ vessel diameter at follow up) and minimal luminal diameter at follow up.

The secondary end points were death, myocardial infarction, need for emergency $C A B G$, and a second intervention involving the previously dilated vessel between the time of randomisation and the follow up angiography performed six months later.

\section{Statistical methods}

The required sample size was based on an assumed restenosis rate of $40 \%$ in the group receiving conventional angioplasty with optional stent implantation and a reduction of that rate by $50 \%$ in the group undergoing direct stenting by an $\alpha$ error of 0.05 and a power of $80 \%$. All primary treatments were compared according to the intention to treat principle. Data are reported as mean (SD). Continuous variables were compared by using the two sided Student's $t$ test for independent samples. The Wilcoxon test was used for categorical variables. A $\chi^{2}$ test or Fisher's exact test (for cells $<5$ ) was used to determine the two tailed significance of associations in $2 \times 2$ tables. Probability values of $p<0.05$ were considered to indicate significance. Data were managed and analysed by the STATISTICA for Windows software package release 5.0 (StatSoft, Tulsa, Oklahoma, USA).

\section{RESULTS}

Between December 1998 and August 1999, 190 patients fulfilled the inclusion criteria and 181 were randomly assigned to either conventional angioplasty with optional stent implantation or direct stent implantation without predilatation. Nine patients declined randomisation because of the repeat angiography at follow up 5.9 (1.6) months (range 1-1 l months) later. One patient in whom angioplasty was not successful underwent elective CABG and did not undergo follow up angiography. In five members of the direct stenting group $(5.6 \%)$ the initial crossing of the target lesion with the stent/ balloon device did not succeed and required predilatation with an angioplasty balloon alone following stent implantation. Finally, 92 patients in the optimal angioplasty group and 89 patients in the direct stenting group completed follow up at 6.5 (2.1) months (range 3-15 months) after the intervention (181 of $190(95.3 \%))$.

\section{Baseline characteristics and clinical follow up}

Table 1 shows selected demographic and clinical characteristics of the two study groups. Baseline characteristics were homogeneously distributed in both study groups. Clinical follow up was obtained for all 181 randomised patients. Forty seven patients in the conventional angioplasty group (51.1\%) required stent implantation to obtain the angiographic criteria for this treatment group. Eight patients underwent repeat angioplasty during hospitalisation (five in the optimal angioplasty group versus three in the primary stenting group, $p=0.38)$. The five patients in the conventional angioplasty group underwent stent implantation-three of them experienced a postprocedural non-fatal Q wave myocardial infarction (maximal CPK $620 \mathrm{U} / \mathrm{l}$ ) and one during the follow up period. Two patients in the direct stenting group suffered a Q 
Table 1 Baseline clinical characteristics

\begin{tabular}{llll}
\hline & $\begin{array}{l}\text { Conventional } \\
\text { angioplasty } \\
\text { (n=92) }\end{array}$ & $\begin{array}{l}\text { Direct } \\
\text { stenting } \\
(\mathbf{n}=89)\end{array}$ & $\mathrm{p}$ Value \\
\hline Mean (SD) age (years) & $61.7(9.9)$ & $61.4(11.8)$ & 0.89 \\
Male sex & $76(82.6 \%)$ & $72(80.1 \%)$ & 0.77 \\
Current smoking status & $23(25.0 \%)$ & $26(29.2 \%)$ & 0.52 \\
Hypertension & $47(51.1 \%)$ & $40(44.9 \%)$ & 0.41 \\
Diabetes mellitus & $11(11.9 \%)$ & $12(13.5 \%)$ & 0.78 \\
Hypercholesterolaemia & $39(42.4 \%)$ & $43(48.3 \%)$ & 0.42 \\
Previous MI & $15(16.3 \%)$ & $19(21.3 \%)$ & 0.39 \\
\hline
\end{tabular}

Current smoking is defined as $\geqslant 10$ cigarettes per day. Hypertension is defined as systolic blood pressure $\geqslant 160 \mathrm{~mm} \mathrm{Hg}$, diastolic blood pressure $\geqslant 90 \mathrm{~mm} \mathrm{Hg}$, or both. Hypercholesterolaemia is defined as cholesterol $>5.2 \mathrm{mmol} / \mathrm{l}$ or high density lipoprotein cholesterol $<0.9 \mathrm{mmol} / \mathrm{I}$ or low density lipoprotein cholesterol $>3.9 \mathrm{mmol} / \mathrm{I}$. MI, myocardial infarction.

Table 2 Procedural complications and clinical outcomes

\begin{tabular}{llll}
\hline & $\begin{array}{l}\text { Conventional } \\
\text { angioplasty } \\
(\mathrm{n}=92)\end{array}$ & $\begin{array}{l}\text { Direct } \\
\text { stenting } \\
(\mathrm{n}=89)\end{array}$ & $\mathrm{p}$ Value \\
\hline Crossover* $_{\text {Sub)acute stent thrombosis }}$ & 0 & $5(5.6 \%)$ & 0.27 \\
Repeat angioplasty & $5(5.4 \%)$ & 0 & 0.13 \\
Q wave MI & $4(4.3 \%)$ & $2(3.4 \%)$ & 0.38 \\
Death & 0 & 0 & 0.36 \\
Elective CABG & $1(1.1 \%)$ & 0 & 1.0 \\
\hline
\end{tabular}

*Crossover from direct stenting to optimal angioplasty. CABG, coronary artery bypass grafting.

wave myocardial infarction $(\mathrm{p}=0.36)$ during hospitalisation (maximal CPK $576 \mathrm{U} / \mathrm{l}$ ). One patient received elective bypass surgery (table 2 ).

\section{Angiographic analysis}

Tables 3 and 4 summarise the qualitative and quantitative angiographic findings. Lesion site and stenosis type were evenly distributed between the two groups. Procedural characteristics (largest balloon size, balloon to artery ratio, and maximal balloon pressure) were similar in both groups. Total inflation time was significantly longer in the optimal angioplasty group (106 (64) seconds in the optional $v 30$ (5) seconds in the direct stenting group, $\mathrm{p}<0.001$ ). There was a significant reduction in late luminal loss in the direct stenting

Table 3 Angiographic findings

\begin{tabular}{|c|c|c|c|}
\hline & $\begin{array}{l}\text { Conventional } \\
\text { angioplasty } \\
(n=92)\end{array}$ & $\begin{array}{l}\text { Direct } \\
\text { stenting } \\
(n=89)\end{array}$ & $\mathrm{p}$ Value \\
\hline Procedural success & $87(94.6 \%)$ & $87(97.8 \%)$ & 0.88 \\
\hline Overall procedure time (min) & $18.7(10.8)$ & $15.2(12.3)$ & 0.043 \\
\hline Fluoroscopy time (min) & $3.5(2.8)$ & $2.3(3.1)$ & 0.0069 \\
\hline Contrast medium (ml) & $73(32)$ & $61(21)$ & 0.0034 \\
\hline Angioplasty catheter & $1.7(1.1)$ & $1.1(1.1)$ & 0.003 \\
\hline \multicolumn{4}{|l|}{ Artery } \\
\hline LAD & $50(54.3 \%)$ & $56(62.9 \%)$ & 0.24 \\
\hline LCX & $9(9.8 \%)$ & $12(13.5 \%)$ & 0.44 \\
\hline RCA & $33(35.9 \%)$ & $21(23.6 \%)$ & 0.07 \\
\hline \multicolumn{4}{|l|}{ Stenosis type } \\
\hline A & $6(6.7 \%)$ & 9 (10.1\%) & 0.38 \\
\hline B1 & $13(13.3 \%)$ & $12(13.5 \%)$ & 0.89 \\
\hline B2 & $53(57.8 \%)$ & $51(57.3 \%)$ & 0.97 \\
\hline $\mathrm{Cl}$ & $16(17.8 \%)$ & $17(19.1 \%)$ & 0.77 \\
\hline $\mathrm{C} 2$ & $4(4.4 \%)$ & $0(0 \%)$ & 0.12 \\
\hline
\end{tabular}

$L A D$, left anterior descending artery; $L C X$, left circumflex artery; RCA, right coronary artery.
Table 4 Results of quantitative coronary angiography

\begin{tabular}{|c|c|c|c|}
\hline & $\begin{array}{l}\text { Conventional } \\
\text { angioplasty } \\
(n=92)\end{array}$ & $\begin{array}{l}\text { Direct } \\
\text { stenting } \\
(n=89)\end{array}$ & $\mathrm{p}$ Value \\
\hline Balloon size (mm) & $3.32(0.30)$ & $3.39(0.27)$ & 0.26 \\
\hline Balloon:artery ratio & 1.02 (0.09) & 1.05 (0.09) & 0.25 \\
\hline $\begin{array}{l}\text { Maximal balloon pressure } \\
\text { (atm) }\end{array}$ & $11.6(3.4)$ & $13.3(1.6)$ & 0.007 \\
\hline Number of stents & $0.6(0.5)$ & $1.0(0.0)$ & $<0.001$ \\
\hline Length of stents $(\mathrm{mm})$ & $17.8(3.6)$ & $15.9(1.7)$ & 0.01 \\
\hline \multicolumn{4}{|l|}{ Reference diameter $(\mathrm{mm})$} \\
\hline Before & $3.18(0.42)$ & $3.22(0.37)$ & 0.69 \\
\hline After & $3.18(0.40)$ & $3.17(0.33)$ & 0.91 \\
\hline At follow up & $3.15(0.39)$ & $3.16(0.40)$ & 0.92 \\
\hline \multicolumn{4}{|c|}{ Minimal luminal diameter $(\mathrm{mm})$} \\
\hline Before & $0.52(0.26)$ & $0.62(0.35)$ & 0.14 \\
\hline After & $2.95(0.60)$ & $3.11(0.54)$ & 0.24 \\
\hline At follow up & $1.87(0.93)$ & $2.56(0.86)$ & 0.002 \\
\hline \multicolumn{4}{|l|}{ Stenosis $(\%)$} \\
\hline Before & $83.4(8.4)$ & 80.5 (10.0) & 0.15 \\
\hline After & $8.9(10.0)$ & $4.8(9.4)$ & 0.06 \\
\hline At follow up & $41.0(28.1)$ & $21.9(22.8)$ & 0.003 \\
\hline Gain (mm) & $2.39(0.67)$ & $2.48(0.64)$ & 0.55 \\
\hline Loss (mm) & $1.19(0.87)$ & $0.62(0.69)$ & 0.004 \\
\hline Net gain (mm) & $1.41(0.93)$ & $1.99(0.92)$ & 0.01 \\
\hline $\begin{array}{l}\text { Diameter stenosis }>50 \% \\
\text { at follow up }\end{array}$ & $28(30.4 \%)$ & $14(15.7 \%)$ & 0.019 \\
\hline
\end{tabular}

group $(1.19(0.87) \mathrm{mm}$ in the optional $v 0.62(0.69) \mathrm{mm}$ in the direct stenting group, $\mathrm{p}=0.004)$ that resulted in a larger minimal luminal diameter ( $1.87(0.93) \mathrm{mm}$ in the optional $v$ $2.56(0.86) \mathrm{mm}$ in the direct stenting group, $\mathrm{p}=0.002)$ and a significant reduction in restenosis rate $(\mathrm{n}=28(30.4 \%) v$ $\mathrm{n}=14(15.7 \%)$, respectively, $\mathrm{p}=0.019$; table 3$)$.

\section{Procedural analysis}

Direct stent implantation resulted in a significantly reduced overall procedural times (15.2 (12.3) minutes in direct $v 18.7$ (10.8) minutes in optional stenting, $p=0.043$ ) and fluoroscopy times $(2.3$ (3.1) minutes in direct $v 3.5$ (2.8) minutes in optional stenting, $p=0.0069)$. The amount of contrast medium used was also reduced in the direct stenting group (61 (21) $\mathrm{ml}$ in direct $v 73$ (32) $\mathrm{ml}$ in optional stenting, $\mathrm{p}=0.0034)$, as was the number of angioplasty catheters used (1.1 (1.1) in direct $v 1.7(1.1)$ in optional stenting, $\mathrm{p}=0.003)$.

\section{DISCUSSION}

Direct stent implantation without predilatation significantly improved long term angiographic follow up after coronary intervention and may have reduced overall procedural costs, although this was not formally analysed.

Since the introduction of coronary artery stents and the confirmation of their efficacy in significantly reducing restenosis and (sub)acute coronary occlusion complicating coronary angioplasty, their use has constantly increased..$^{10-12}$

Ultrasound studies have shown that the improvement of minimal luminal diameter resulting in a reduced restenosis rate was conditioned by the enlargement of the target vessel by stents. ${ }^{13}$ The problem of smooth muscle cell proliferation after coronary angioplasty with or without coronary stent implantation remains unresolved. Therefore, further efforts were made to reduce this biological problem by drug treatment or brachytherapy. ${ }^{14-16}$

The results of drug treatment trials have shown no effects on restenosis. Even the use of the new glycoprotein IIb/IIIa receptor antagonists had no influence on restenosis. ${ }^{17}$ Other strategies are not yet practicable for use in daily clinical practice and are limited to a very small, well selected group of patients. 


\begin{tabular}{|c|c|c|c|}
\hline & $\begin{array}{l}\text { Conventional } \\
\text { angioplasty } \\
\text { (+ stent) }(n=47)\end{array}$ & $\begin{array}{l}\text { Direct } \\
\text { stenting } \\
(n=89)\end{array}$ & p Value \\
\hline Balloon size $(\mathrm{mm})$ & $3.32(0.31)$ & $3.39(0.27)$ & 0.35 \\
\hline Balloon:artery ratio & $1.00(0.09)$ & $1.05(0.09)$ & 0.068 \\
\hline $\begin{array}{l}\text { Maximal balloon } \\
\text { pressure (atm) }\end{array}$ & $13.7(1.2)$ & $13.3(1.6)$ & 0.23 \\
\hline Number of stents & $1.0(0.0)$ & $1.0(0.0)$ & 1 \\
\hline Length of stents $(\mathrm{mm})$ & $17.8(3.6)$ & $15.9(1.7)$ & 0.012 \\
\hline \multicolumn{4}{|c|}{ Reference diameter $(\mathrm{mm})$} \\
\hline Before & $3.30(0.45)$ & $3.22(0.37)$ & 0.46 \\
\hline After & $3.28(0.44)$ & $3.17(0.33)$ & 0.29 \\
\hline At follow up & $3.19(0.44)$ & $3.16(0.40)$ & 0.77 \\
\hline \multicolumn{4}{|c|}{ Minimal luminal diameter $(\mathrm{mm})$} \\
\hline Before & $0.47(0.23)$ & $0.62(0.35)$ & 0.035 \\
\hline After & $3.14(0.61)$ & $3.11(0.54)$ & 0.86 \\
\hline At follow up & $1.93(0.98)$ & $2.56(0.86)$ & 0.031 \\
\hline \multicolumn{4}{|l|}{ Stenosis (\%) } \\
\hline Before & $85.3(7.8)$ & $80.5(10.0)$ & 0.022 \\
\hline After & $5.9(8.4)$ & $4.8(9.4)$ & 0.74 \\
\hline At follow up & $39.5(30.5)$ & $21.9(22.8)$ & 0.037 \\
\hline Gain (mm) & $2.64(0.64)$ & $2.48(0.64)$ & 0.31 \\
\hline Loss (mm) & $1.22(0.90)$ & $0.62(0.69)$ & 0.018 \\
\hline Net gain $(\mathrm{mm})$ & $1.53(0.93)$ & $1.99(0.92)$ & 0.15 \\
\hline $\begin{array}{l}\text { Diameter stenosis } \\
\quad>50 \% \text { at follow up }\end{array}$ & $13(28.6 \%)$ & $14(15.7 \%)$ & 0.011 \\
\hline
\end{tabular}

The idea of reducing the extent of vessel wall injury and thereby of reducing restenosis is not new. Previous studies failed to show a significant effect of this principle on long term follow up after coronary angioplasty. Recently published data showed only a non-significant trend in the reduction of restenosis following coronary angioplasty by gradually increased balloon expansion as compared with conventional, quick balloon inflation. ${ }^{18}$ Although the dilated segment was not evaluated by ultrasound, late luminal loss was used to calculate the extent of smooth muscle cell proliferation.

The main variable found to determine the development of restenosis was the minimal luminal diameter after intervention. ${ }^{19}$ Therefore, it seems opportune to reach this objective within the meaning of the words "bigger is better" ${ }^{20}$ As described in BENESTENT (Belgium Netherlands stent study) and STRESS (stent restenosis study), the target vessel enlargement achieved with coronary artery stents significantly reduced the restenosis rate. ${ }^{10}{ }^{11}$ On the other hand, this vessel enlargement by stent implantation resulted in a significant increase of late luminal loss in the stent group. Therefore, conventional angioplasty with optional stent implantation followed the bigger is better principle. Table 4 shows a late luminal loss and a restenosis rate that is comparable with the long term results published in previous trials. $^{21} 22$

In the direct stent implantation group the target lesion was crossed with a stent/balloon device, combining two treatment strategies-coronary angioplasty and coronary stent implantation. Recently published investigations confirmed the feasibility and efficacy of this approach in selected lesion morphology, but a comparison with an angioplasty procedure as used routinely by interventional cardiologists was not yet available. ${ }^{4}$

We found a significant reduction in late luminal loss, resulting in an increased net gain, larger minimal luminal diameter, and lower restenosis rate at follow up by direct stent implantation without predilatation. The assumption that these effects were caused by reduced vessel wall injury was confirmed by the fact that these differences remained significant even when we compared direct stenting with the subgroup of patients from the conventional angioplasty group who received stents to achieve an optimal angioplasty result (table 5).

Although they were not end points, overall procedural and fluoroscopy times, as well as the amount of contrast medium and the number of angioplasty catheters used, were significantly reduced in the direct stenting group, helping to reduce the costs of this procedure.

\section{Complications}

(Sub)acute vessel occlusion complicating coronary intervention occurred more often in the conventional angioplasty group ( $3 v 0$ in the direct stenting group, $\mathrm{p}=0.13$ ) and did not differ from previous published data. ${ }^{22}$ The incidence of postprocedural and overall myocardial infarction also differed between the groups but these differences were not significant (table 3). No patient underwent acute coronary bypass surgery. One patient in the optimal angioplasty group underwent elective bypass surgery for failed angioplasty. No patient died during follow up. Five patients in the direct stenting group were reallocated to the conventional angioplasty group because the crossing of the target lesion with the stent/balloon device did not succeed, making predilatation with a balloon catheter necessary.

The lower incidence of clinical events in the direct stent group was caused by the routine use of coronary stents and in our opinion by the reduction of vessel wall injury.

\section{Limitation of the study}

The present trial was a single centre study representing the experience of only one hospital. Therefore, a bias cannot be excluded as to which patients seemed suitable for direct stent implantation. Patients with acute coronary syndrome and totally occluded vessels were excluded from this trial. The positive effects of direct stent implantation without predilatation could therefore be confirmed only in vessels suitable for direct stenting: fairly short, de novo lesions in vessels with a diameter $>2.75 \mathrm{~mm}$.

The sample size was too small and not calculated to allow subgroup analysis; therefore, the differences between subgroups were not significant and indicate only an angiographic, not a clinical, benefit. This was biased by lesion selection because in the conventional angioplasty group only patients with unfavourable lesion morphology underwent stent implantation.

\section{Conclusion}

Direct stent implantation without predilatation significantly improves minimal luminal diameter and reduces late luminal loss and restenosis rate at follow up after coronary intervention in de novo lesions in arteries $>2.75 \mathrm{~mm}$, as compared with conventional angioplasty with optional stent implantation. The incidence of complications was similar in both groups except for the incidence of crossover from the direct stenting group to the conventional angioplasty group because of failure in crossing the target lesion with the stent delivery device.

The procedure may help to reduce the cost of coronary interventions by reducing overall procedure and fluoroscopy times, the amount of contrast medium used, and the number of angiography cathethers required.

\section{Authors' affiliations}

S Miketic, J Carlsson, U Tebbe, Department of Cardiology, Klinikum Lippe-GmbH, Detmold, Germany

\section{REFERENCES}

1 Leon MB, Popma JJ, Mintz GS, et al. An overview of US coronary stent trials. Semin Interv Cardiol 1996;1:247-54. 
2 Pentousis D, Guerin Y, Funck F, et al. Direct stent implantation without predilatation using the Multilink stent. Am J Cardiol 1998;82:1437-40.

3 Hamon $M$, Richardeau $Y$, Lécluse $E$, et al. Direct coronary stenting without predilatation in acute coronary syndromes. Am Heart 1999;138:55-9.

4 Briguori C, Sheiban I, De Gregorio J, et al. Direct coronary stenting without predilatation. J Am Coll Cardiol 1999;34:1910-5.

5 Ojha $M$. Wall shear stress temporal gradient and anastomotic intimal hyperplasia. Circ Res 1994;74:1227-31.

6 Figulla HR, Mudra H, Reifart N, et al. Direct coronary stenting without predilatation: a new therapeutic approach with a special balloon catheter design. Cathet Cardiovasc Diagn 1998;43:245-52.

7 Wilson SH, Berger PB, Mathew V, et al. Immediate and late outcome after direct stent implantation without balloon predilatation. J Am Coll Cardiol 2000:35:937-43.

8 Ryan TJ, Baumann WB, Kennedy JW, et al. Guidelines for percutaneous transluminal coronary angioplasty. J Am Coll Cardiol 1993;22:203354.

9 Reiber LHC, Schiemanck LR, van der Zwet PM, et al. QCA: technical update 1995. In: de Feyter PJ, di Mario C, Serruys PW, eds. Quantitative coronary imaging. Rotterdam: Barjesteh, Meeuwes, 1995:9-27.

10 Serruys PW, de Jaegere P, Kiemeneij F, et al, for the Benestent Study Group. A comparison of balloon-expandable stent implantation with balloon angioplasty in patients with coronary artery disease. N Engl J Med 1994:331:489-95.

11 Fischman DL, Leon MB, Baim DS, et al, for the Stent Restenosis Study Investigators. A randomized comparison of coronary-stent placement and balloon angioplasty in the treatment of coronary artery disease. N Engl Med 1994;331:496-501.

12 Jacobs AK. Coronary stents: have they fulfilled their promise? N Engl J Med 1999;341:2005-6.
13 Albiero $\mathbf{R}$, Rau T, Schlüter $M$, et al. Comparison of immediate and intermediate-term results of intravascular ultrasound versus angiography-guided Palmaz-Schatz stent implantation in matched lesions. Circulation 1997;96:2997-3005.

14 Serruys PW, Foley DP, Höfling B, et al. Carvedilol for preventing of restenosis after directional coronary atherectomy. Circulation $2000 \cdot 101: 1512-8$

15 Jorgensen B, Simonsen S, Endresen K, et al. Restenosis and clinical outcome in patients treated with amlodipine after angioplasty: results from the coronary angioplasty amlodipine restenosis study (CAPARES). Am Coll Cardiol 2000;35:592-9

16 Teirstein P, Massullo V, Jani S, et al. Three-year clinical and angiographic follow-up after intracoronary radiation. Circulation 2000;101:360-5.

17 Topol EJ, Califf RM, Weisman HF, et al, on behalf of the EPIC investigators. Randomised trial of coronary intervention with antibody against platelet llb/llla integrin for reduction of clinical restenosis: results at six months. Lancet 1994;343:881-6.

18 Miketic S, Carlsson J, Tebbe U. Influence of gradually increased slow balloon inflation on restenosis after coronary angioplasty. Am Heart J 1998:135:709-13

19 Kuntz RE, Safian RD, Carrozza JP, et al. The importance of acute lumina diameter in determining restenosis after coronary atherectomy or stenting. Circulation 1992;86:1827-35

20 Meier B. How to treat small coronary vessels with angioplasty. Heart 1998;79:215-6.

21 Dangas G, Ambrose JA, Rehmann D, et al. Balloon optimization versus stent study (BOSS): provisional stenting and early recoil after balloon angioplasty. Am J Cardiol 2000;85:957-61.

22 Kimura T, Yokio $\mathrm{H}$, Nakagawa $\mathrm{Y}$, et al. Three-year follow-up after implantation of metalic coronary-artery stents. N Engl J Med 1996;334:561-6.

\section{IMAGES IN CARDIOLOGY}

\section{Atrio-auricular mechanical dissociation following electrical cardioversion for atrial fibrillation}

$A^{\text {m }}$ rial stunning occurs in about $20 \%$ of patients cardioverted for atrial fibrillation, being characterised by absence of mechanical activity of the atria despite normal activation. We report an unusual example of mechanical dissociation between the left atrium and the appendage. A 67 year old patient underwent DC shock for permanent atrial fibrillation $(200 \mathrm{~J})$; the precardioversion transoesophageal echocardiogram (below) shows relatively high velocity emptying (E) and filling (F) waves at the auricular level (LA, left atrium, LAA, left atrial appendage, LV, left ventricle). The images recorded 24 hours following sinus rhythm restoration (upper panel, right) demonstrate a paradoxical auricular flow during atrial systole; in coincidence with the $\mathrm{P}$ wave, thus, a filling wave occurs (arrow) instead of the expected emptying wave. In other words, the left atrial appendage does not contract simultaneously with the atrium during systole, so that some blood is forced from the atrial cavity into the appendage. Three weeks later, in contrast (lower panel, right), normal auricular flow is restored, as demonstrated by the expected emptying wave (arrow) simultaneous to the $\mathrm{P}$ wave.

S Careri

$M$ P Calabrò

S Di Rosa

scipione2@interfree.it

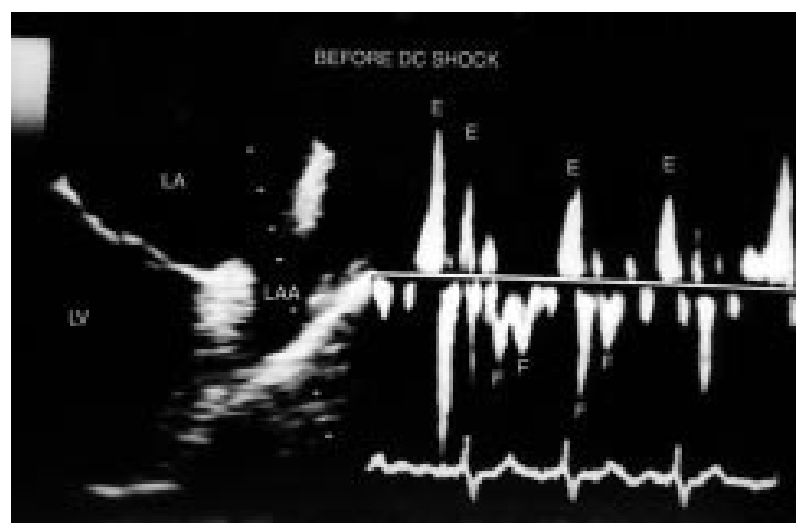

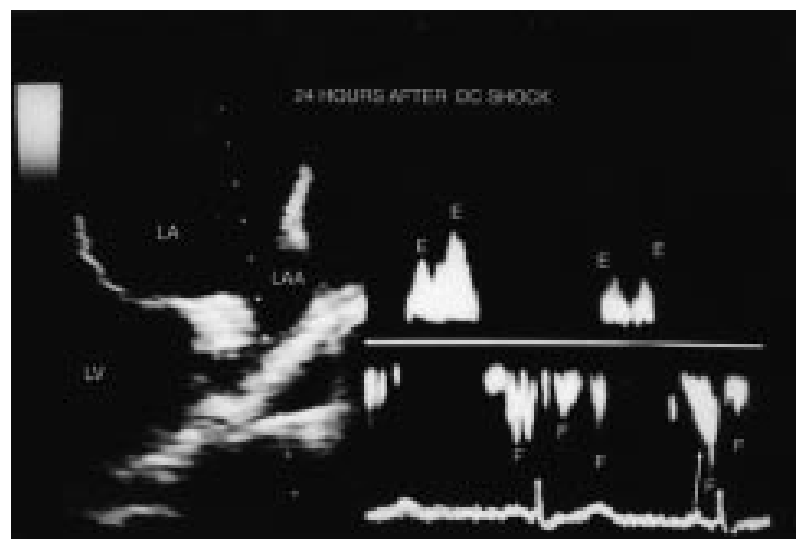

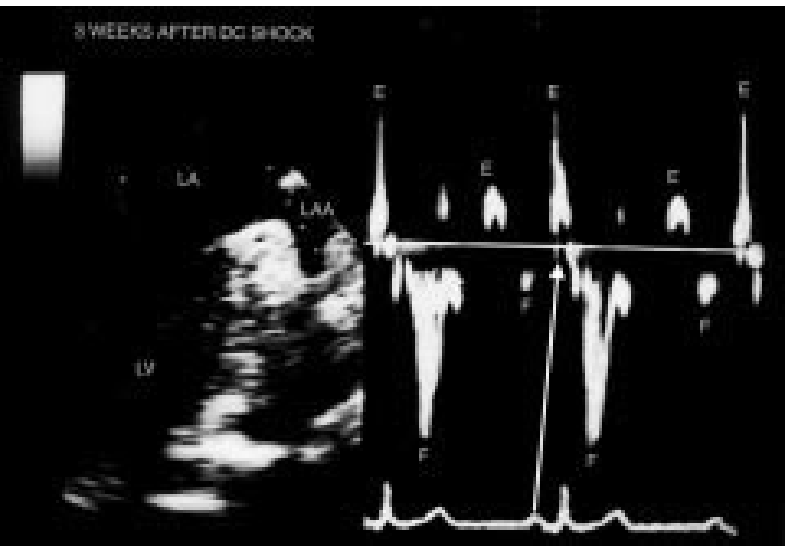

